\title{
EUS-Guided Celiac Plexus Interventions
}

\author{
Zubin Dev Sharma ${ }^{1}$ Rinkesh Kumar Bansal ${ }^{2} \quad$ Rajesh Puri ${ }^{1}$
}

\author{
${ }^{1}$ Institute of Digestive and Hepatobiliary Sciences, Medanta, The \\ Medicity, Gurgaon, Haryana, India \\ 2Department of Gastroenterology \& Hepatology, Fortis Memorial \\ Research Institute, Gurgaon, Haryana, India
}

\begin{abstract}
Address for correspondence Rajesh Puri, DNB (Gastro), Department of Interventional Gastroenterology, Institute of Digestive and Hepatobiliary Sciences, Medanta, the Medicity, Gurgaon, Haryana, India (e-mail: purirajesh1969@gmail.com).
\end{abstract}

J Digest Endosc:2020;11:177-181

\begin{abstract}
Keywords

- EUS

- EUS-CPB

- EUS-CPN

- EUS-RFA

Endoscopic ultrasound (EUS)-guided celiac plexus intervention comprises EUS-guided celiac plexus block (EUS-CPB) and EUS-guided celiac plexus neurolysis (EUS-CPN). EUS-CPB and EUS-CPN are one of the used methods of pain management in patients of pancreatic cancer and chronic pancreatitis, respectively, along with medical therapy. Both the procedures are associated with minimal complications and improves quality of life. The role of EUS-guided celiac plexus intervention is increasing in gall bladder carcinoma for pain control.
\end{abstract}

\section{Introduction}

Pancreatic cancer and chronic pancreatitis are the two conditions associated with significant pain; management of pain is often less rewarding and requires multimodality approach.

Pancreatic cancer is widely prevalent and associated with very poor prognosis. Though India has lower incidence of pancreatic cancer than western countries, its rate is on rising trends. In India, the incidence rate varies from 0.5 to $2.4 / 100,000$ persons per year among women to 0.2 to $1.8 / 100,000$ persons per year among men. There is estimate that in 2020, around 15,000 new cases of pancreatic cancer would be diagnosed in India. ${ }^{1,2}$ The overall 5 years survival remains poor at $7.7 \%$; $29.3 \%$ for localized and $2.6 \%$ for metastatic disease. Majority of pancreatic cancer patients complain of pain during the course of their illness. Some authors considered occurrence of pain as a marker of unresectability on imaging. ${ }^{3}$

Chronic pancreatitis is a condition that is characterized by irreversible damage to the pancreas that eventually leads to pain and/or exocrine and endocrine insufficiency. It is a major health problem worldwide and is associated with considerable morbidity. Commonest cause is idiopathic; though, alcohol is one the most common causes worldwide. In a prospective nationwide study of 1,086 Indian patients with chronic pancreatitis, pain was present in 971 subjects (94.0\%). Of the 85 subjects who were reported to have undergone surgery, relief of pain was reported in 31 subjects (36.5\%). ${ }^{4}$
Pain is a major complaint, and the medical management of pain in these conditions is usually inadequate and disappointing. Pain severity also correlates with decreased survival. ${ }^{5}$ Opiate-based systemic analgesic therapy is often insufficient, requiring doses to be increased with the times that is often associated with side-effects such as sedation and constipation among others.

If patients have refractory pain or cannot tolerate increasing amounts of opioid medications, EUS-guided celiac plexus intervention play a very important role.

EUS-guided celiac plexus interventions can be divided into celiac plexus block (EUS-CPB) and celiac plexus neurolysis (EUS-CPN). EUS-CPB involves the injection of an anesthetic, with or without steroids. This leads to temporary pain relief and nonpermanent destruction of celiac plexus. EUSCPN involves obliteration of the celiac plexus by injection of a neurolytic agent (with or without an anesthetic agent such as bupivacaine). Neurolytic agents used during EUS-CPN induce a local inflammatory reaction, followed by fibrosis during the healing process.

\section{History of CPB and CPN}

Celiac plexus interventions were initially described in 1914 as an intra-operative ablative procedure. Subsequently, it has been done under fluoroscopic, computed tomography (CT) guided, ultrasound (USG) guided or EUS guided. EUSguided (CPN) was described by Faigel et al and Wiersema 
in $1996 .{ }^{6}$ The first case of EUS-guided (CPB) in pain associated with chronic pancreatitis was done in 1999 by Gress. ${ }^{7}$ After these initial descriptions, many studies have been conducted for both CPN and CPB, comparing its efficacy, longevity, and ease of doing in comparison to CT-guided procedures.

The advantage of the EUS approach is the control of the needle above or lateral to the celiac trunk and use of Doppler to visualize vessel interposition.

\section{Clinical Anatomy and EUS Localization}

The celiac plexus carries pain fibers from upper abdominal viscera, mesentery, small and large bowel proximal to the transverse colon. The celiac plexus consists of network of connected para-aortic ganglia. It consists of celiac, superior mesenteric, and renal ganglia located at roots of celiac artery, superior mesenteric artery (SMA) and renal artery. The celiac plexus is a hub for parasympathetic, sympathetic, and noci-

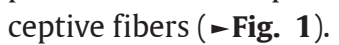

The celiac plexus lies within the retroperitoneal space posterior to the stomach, embedded in loose areolar tissue close to the celiac axis anterolateral to the aorta. There is considerable variability in size, number, and position of ganglia.

EUS localization is important for adequate intervention. The celiac plexus is usually not seen as a discrete structure but its location is determined relative to the celiac artery. Rarely ganglia can be seen as discrete structures. These are usually seen anterior to the aorta (slightly to the left), cephalad to the celiac artery take-off and medial to the left adrenal gland. They appear as hypoechoic, oblong, or lobulated structures, often with irregular edges and usually contain hyperechoic foci or strands. ${ }^{8,9}$

\section{Indications}

The indication of EUS-CPB and EUS-CPN is intractable abdominal pain in the setting of pancreatic cancer or chronic pancreatitis that is not managed by conservative methods. EUS-CPB is used for patients of chronic pancreatitis who fail conservative treatment plans. EUS-CPN, that is, complete obliteration of celiac plexus using neurolytic agents is to be used in pancreatic malignancies only. National Comprehensive Cancer Network (NCCN) guidelines also recommend use of CPN for intractable pain in pancreatic cancer. ${ }^{10} \mathrm{CPN}$ is especially beneficial when patients cannot tolerate adverse effects of opioid therapy, such as drowsiness, somnolence, confusion, delirium, dry mouth, anorexia, constipation, nausea, and vomiting, or if maximum achievable dose of analgesics is achieved. Many believe that EUS-CPN should be considered early in pancreatic cancer due to the fact that in advanced malignancy, the pain usually becomes multifactorial and there is centralization of the pain. However, a study published by de Oliveira et al in 2004 showed no difference in pain reduction in early versus late neurolytic plexus block in 25 patients with intra-abdominal cancer. In chronic pancreatitis, World Health Organization (WHO) pain ladder should be followed and EUSCPB to be considered when conventional methods fail.

\section{Contraindications}

Contraindications for CPB or neurolysis are: patients with coagulopathy, local/intra-abdominal infection and sepsis, bowel obstruction, patients on disulfiram therapy for alcohol abuse, patients with physical dependence and drug-seeking behavior, thrombocytopenia (platelets $<50,000$ ), and an uncooperative patient.

Patients taking disulfiram are not good candidates, because they can get tachycardia, nausea, vomiting, and headache because of accumulation of acetaldehyde, as the alcohol is not broken down easily.

\section{Technique of EUS-Guided Intervention}

The patient is usually in the left lateral position. Preprocedural and procedural hydration are a must to prevent hypotension. During the procedure, the patient should be continually monitored for hypotension.

Linear EUS Scope is used for procedure. A 22-gauge EUS needle is frequently used. There have been specific needles designed for EUS-CPB and EUS-CPN. Cook Medical designed a 20-gauge needle that differs from other EUS needles in that it has a sharp, solid and conical tip with side holes for adequate delivery of agent radially. There have been no head to head studies comparing this needle with standard EUS-FNA (fine needle aspiration) needles. Some centers also use 19-gauge needle. For anesthesia, propofol or midazolam can be used. Some endosonologist recommend antibiotic prophylaxis, which is thought to help avoid formation of retroperitoneal abscess (-Fig. 2).

The procedure can be done in three ways. Injection of neurolysis/blocking agent can be done at base of the celiac trunk (unilateral approach), or on the sides of the celiac trunk (bilateral approach). If ganglia are visible, then direct ganglia injection also can be done. ${ }^{11,12}$

For EUS-CPN, absolute alcohol is used. $0.25 \%$ bupivacaine may be injected just before injecting 10 to $20 \mathrm{~mL}$ absolute alcohol.

EUS-CPB is generally performed with the unilateral approach. For unilateral approach, $20 \mathrm{~mL}$ of $0.25 \%$ bupivacaine with $80 \mathrm{mg}$ triamcinolone is injected on one side. Around $20 \mathrm{ml}$ of $0.25 \%$ bupivacaine is followed by $40 \mathrm{mg}$ of triamcinolone on each side of the celiac plexus in bilateral approach. The total amount remains same as in unilateral approach, that is, $20 \mathrm{~mL}$.

\section{The Central or Unilateral Approach}

In the central approach, the aorta is first identified in the longitudinal axis on the posterior wall of the stomach, below gastroesophageal junction. Aorta usually comes into imaging with clockwise rotation from the liver hilum. Then the origin of celiac artery is identified by manipulation of the scope and little forward push. After this, a 22-gauge needle is passed to just above the origin of celiac artery. Always flush the needle with $3 \mathrm{~mL}$ of normal saline before injection to flush out any tissue lodged in needle during passing. Aspiration is mandatory to rule out inadvertent puncture of blood vessel. This is 
a critical step in this process, as direct injection into a blood vessel can lead to significant complications.

Following this, the agent of choice (absolute alcohol or bupivacaine with triamcinolone) is injected. If using absolute alcohol, an echogenic cloud can be obtained. In case echogenic cloud is not visible, the position of needle should be rechecked and empirical injection of more agent should not be attempted. Before withdrawing the needle, it should be flushed with $3 \mathrm{~mL}$ normal saline to prevent seeding of the needle track with alcohol, which may produce transient severe post-procedure pain.

\section{Bilateral Technique}

Following identification of celiac artery, the linear echo endoscope is rotated clockwise till both celiac artery and SMA are no longer visible. The needle is then advanced to inject in this area, lateral to point where SMA originates. After this the needle is withdrawn, and echo endoscope is rotated in counter clockwise rotation until again celiac artery and SMA are not seen. The agent is then injected in this right lateral base. Total amount of injected agent usually remains the same.

\section{EUS-Guided Celiac Ganglia Neurolysis (EUS-CGN)}

Levy et $\mathrm{al}^{11}$ developed this technique. The celiac ganglia are identified in between aorta and the left adrenal gland and then ethanol is directly injected into the ganglia. The CG are hypoechoic caterpillar-like or small and nodular structures. CG are injected with absolute alcohol till the targeted ganglion becomes hyperechoic and difficult to visualize.

\section{Evidence for CPN and CPB}

\section{Efficacy of CPN and CPB}

In the original description of Wiersema and Wiersema et a ${ }^{12}$ in $1996, \sim 80$ to $88 \%$ patients reported long lasting pain relief with CPN and 81 to $90 \%$ patients reported lower use of pain medication.

There have been two meta-analyses that evaluated the pain relief for both EUS-CPN and EUS-CPB. The first was meta-analysis by Puli et $\mathrm{al}^{13}$ in which 8 studies $(n=283)$ for EUS-CPN for pain due to pancreatic cancer and nine studies for chronic pancreatitis $(n=376)$ were taken. In pancreatic cancer, the pooled pain relief was around $80.12 \%$ and in chronic pancreatitis it was at $59.45 \%$. They concluded with saying that better technique or agents are needed for pain relief in chronic pancreatitis.

In another meta-analysis, by Kaufman et al $^{14}$ a total of 9 studies were included in the final analysis. For chronic pancreatitis, 6 relevant studies $(n=221)$ were identified and EUS-CPB was effective in alleviating abdominal pain in $51.46 \%$ of patients. For pancreatic cancer, 5 relevant studies $(n=119)$ were identified and EUS-CPN was effective in alleviating abdominal pain in $72.54 \%$ of patients.

For CPB in chronic pancreatitis, the average length of pain relief was around 3 months, hence it was recommended that $\mathrm{CPB}$ was considered as temporizing measure in pain relief in chronic pancreatitis. Some studies ${ }^{15}$ have shown that repeated $\mathrm{CPB}$ can be safe choice and response to first CPB is a predictor of responses to subsequent sessions of blocks.

\section{CGN versus CPN}

The initial report on EUS-CGN by Levy et al ${ }^{11}$ showed a pain relief in $94 \%$ pancreatic cancer patients treated by EUS-CGN. In the case of chronic pancreatitis, $80 \%$ of those who received alcohol injections reported pain relief versus 38\% of those who received steroid injections. This study was criticized for having small sample size. Ascunce ${ }^{16}$ did a multivariate analysis to determine the predictive factors of response in which EUS-CGN was performed when the CG were visible by EUS and bilateral EUS-CPN was performed. Identification of celiac ganglion was associated with better results as compared with bilateral CPN. Another study was done by Levy et $\mathrm{al}^{17}$ in 2018 , which was a randomized trial comparing combined celiac ganglia and plexus neurolysis versus plexus neurolysis alone. This was done in 60 and 50 patients, respectively, in both the groups. Primary outcomes were pain control, quality of life at 12 weeks and overall survival. Rate of pain response to CGN was $46.2 \%$ and CPN was $40.4 \%$, which was statistically nonsignificant. Additionally, survival time was significantly shorter for patients undergoing CGN as compared with CPN.

\section{EUS CPB versus CT-Guided CPB}

In a small study by Gress et al, 7 post-procedure pain reduction was in 50 versus $25 \%$ of patients for EUS-CPB versus CT guided. Persistent pain relief was also more in EUS-CPB. In an RCT by Santosh et al, ${ }^{18}$ initial pain relief was seen in $70 \%$ of patients in comparison with $30 \%$ for CT guided.

\section{Bilateral versus Unilateral CPN}

Bilateral EUS-CPN gives higher pain relief, but technical feasibility and operator comfort justify central injection as a viable alternative. In a prospective randomized control trial, which included around 160 patients, Sahai et $\mathrm{al}^{19}$ showed that bilateral technique showed significantly more pain relief versus unilateral one, that is, 70 versus $45 \%$. This value was highly significant at day 7 of treatment. The meta-analysis by Puli et $\mathrm{a}^{13}$ also showed that in case of bilateral injection, the proportion of patients with pain relief was 84.54 versus $45.99 \%$ with unilateral.

\section{Impact of EUS CPB or CGN on Survival in Pancreatic Cancer}

Levy et al in 2015 in a large retrospective study of 417 patients showed that celiac neurolysis was an independent predictor of shortened survival in pancreatic cancer patients. The study, however, could not identify that this shortened survival was due to neurolysis or patient or disease characteristics. In another study done by Tak et al in 2017, CPN did not affect survival of patients with unresectable pancreatic malignancy, but it was a retrospective study. In a further study done by Levy et $\mathrm{al}^{17}$ in 2018 , they found CGN to shorten survival with no differences in quality of life as compared with CPN. Hence, they recommended reassessment of role of CGN in pain management for unresectable pancreatic cancer. 


\section{Complications}

Commonly seen complications include transient diarrhea, Hypotension, transient increase in pain amongst others. Overall, the complication rate is less than $10 \%$, although minor complications have been reported to the extent of $30 \%$ in some studies. A review study of 20 publications comprising 1,142 patients showed that complications occurred in $7 \%$ of 481 EUS-CPB procedures and in $21 \%$ of 661 EUS-CPN procedures. ${ }^{20}$ Rare complications include infective like retroperitoneal abscess, bleeding, or pseudoaneurysm formation, ischemic injuries. Extremely rarely paraplegia has been reported. In a case series of 2,730 CPN procedures, major complications of neurological deficits were limited to four cases $(<0.2 \%)$ All four cases had permanent paraplegia, while three of the four cases had loss of anal and bladder sphincter function as well..$^{21,22}$ Antibiotic prophylaxis is recommended when doing $\mathrm{CPB}$ in most patients.

\section{Recent Updates}

Recently, a new modality, that is, EUS-guided radiofrequency ablation (RFA) has been studied as palliative treatment option for pancreatic neoplasms. A randomized trial was done to compare the effectiveness of EUS-CPN $(n=14)$ and EUS-RFA $(n=12)$ for palliation of pain in patients with locally advanced or metastatic pancreatic cancer. EUS-RFA was performed using a $1 \mathrm{~F}$ monopolar probe passed via a 19-gauge FNA needle, by targeting the area of celiac plexus or visualized ganglia. They found that EUS-RFA provided more pain relief and improved the quality of life for patients with pancreatic cancer when compared with EUS-CPN. ${ }^{23}$

The role of EUS-CPN was also evaluated prospectively in patients with unresectable gall bladder carcinoma (GBC) with upper abdominal pain, not responding to usual drugs. Total 21 patients were enrolled. There was a significant reduction in daily requirement of analgesics in all the patients at 2 and 4 weeks compared with baseline $(p<0.001)$; however, at week 8 , there was no significant reduction in analgesic dose. So, they concluded that EUS-CPN has been highly technically feasible, quite effective, and safe in most patients with GBC. ${ }^{24}$

Both the above studies appear promising, however, large prospective randomize controlled trials will be required in future.

Various series have also used iodine-125 seeds for EUSguided celiac ganglion irradiation..$^{25}$ This did not increase the survival but did have reduction in pain scores. This has not been approved yet and further studies are still needed.

Kanno et $\mathrm{al}^{26}$ did a prospective randomized trial comparing EUS-guided neurolysis versus medications alone in unresectable pancreatic cancer in the oxycodone/fentanyl era. The study comprised two arms. A total of 24 patients underwent EUS-guided neurolysis and 22 patients formed the control arm. No severe procedural side-effects were reported. Average pain scores decreased in both the groups. The difference was not statistically significant at week 1,2 , 8 , and 12 . They concluded that EUS-CPN in pancreatic cancer pain did not appear to improve pain, quality of life, and

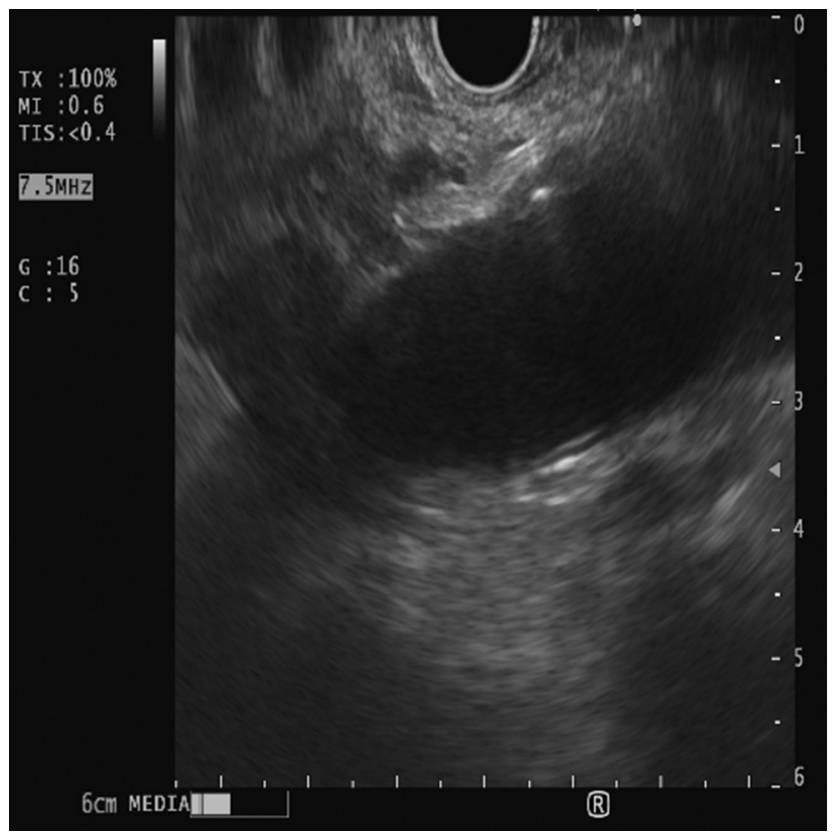

Fig. 1 Endoscopic ultrasound image showing celiac plexus ganglia.

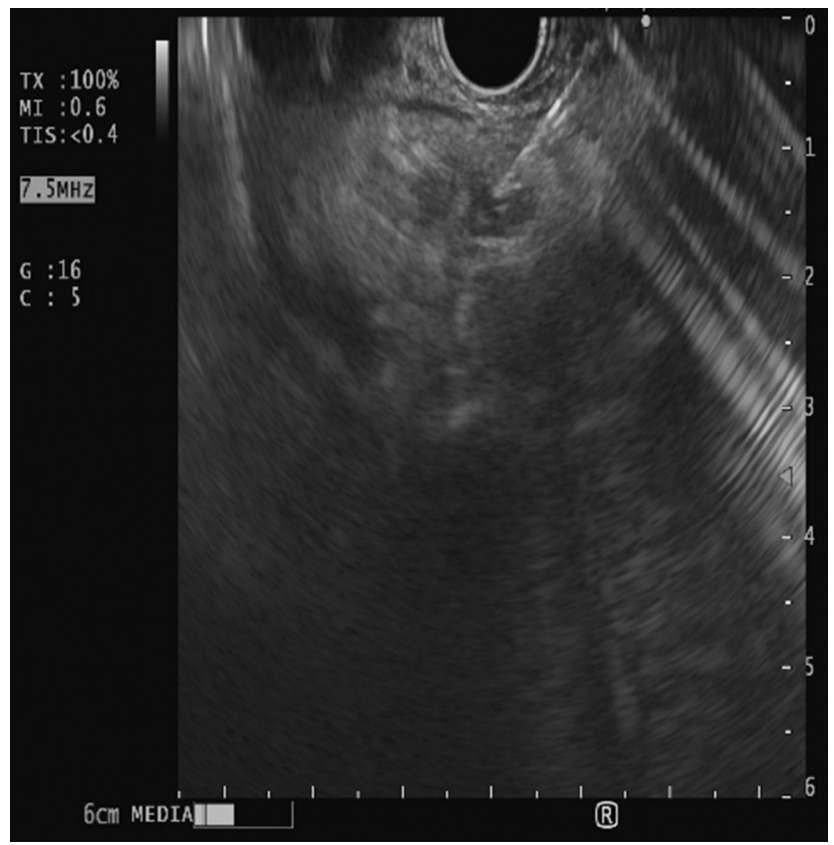

Fig. 2 Endoscopic ultrasound image of celiac ganglia neurolysis with evidence of needle.

opioid consumption. This study was questioned for its lack of effectiveness in its EUS-CPN arm and further data are awaited.

\section{Conclusion}

EUS-CPN and EUS-CPB are safe and effective means of pain management in patients of pancreatic cancer and chronic pancreatitis. Bilateral technique gives better pain control but 
is technically demanding. Complication rates are low. Early CPN can help in better pain management in patients of pancreatic cancer and lead to better quality of life.

\section{Conflict of Interest}

None.

\section{References}

1 Dhir V, Mohandas KM. Epidemiology of digestive tract cancers in India IV. Gall bladder and pancreas. Indian J Gastroenterol 1999;18(1):24-28

2 Takiar R, Nadayil D, Nandakumar A. Projections of number of cancer cases in India (2010-2020) by cancer groups. Asian Pac J Cancer Prev 2010;11(4):1045-1049

3 Yan BM, Myers RP. Neurolytic celiac plexus block for pain control in unresectable pancreatic cancer. Am J Gastroenterol 2007;102(2):430-438

4 Balakrishnan V, Unnikrishnan AG, Thomas V, et al. Chronic pancreatitis. A prospective nationwide study of 1,086 subjects from India. JOP 2008;9(5):593-600

5 Koulouris AI, Banim P, Hart AR. Pain in patients with pancreatic cancer: Prevalence, mechanisms, management and future developments. Dig Dis Sci 2017;62(4):861-870

6 Faigel DO, Veloso KM, Long WB, Kochman ML. Endosonographyguided celiac plexus injection for abdominal pain due to chronic pancreatitis. Am J Gastroenterol 1996;91(8):1675

7 Gress F, Schmitt C, Sherman S, Ikenberry S, Lehman G. A prospective randomized comparison of endoscopic ultrasound- and computed tomography-guided celiac plexus block for managing chronic pancreatitis pain. Am J Gastroenterol 1999;94(4):900-905

8 Gerke H, Silva RG Jr, Shamoun D, Johnson CJ, Jensen CS. EUS characteristics of celiac ganglia with cytologic and histologic confirmation. Gastrointest Endosc 2006;64(1):35-39

9 Ha TI, Kim GH, Kang DH, Song GA, Kim S, Lee JW. Detection of celiac ganglia with radial scanning endoscopic ultrasonography. Korean J Intern Med (Korean Assoc Intern Med) 2008;23(1):5-8

10 Caraceni A, Portenoy RK. Pain management in patients with pancreatic carcinoma. Cancer 1996;78(3(Suppl):639-653

11 Levy MJ, Topazian MD, Wiersema MJ, et al. Initial evaluation of the efficacy and safety of endoscopic ultrasound-guided direct Ganglia neurolysis and block. Am J Gastroenterol 2008;103(1):98-103

12 Wiersema MJ, Wiersema LM. Endosonography-guided celiac plexus neurolysis. Gastrointest Endosc 1996;44(6):656-662

13 Puli SR, Reddy JB, Bechtold ML, Antillon MR, Brugge WR. EUS-guided celiac plexus neurolysis for pain due to chronic pancreatitis or pancreatic cancer pain: a meta-analysis and systematic review. Dig Dis Sci 2009;54(11):2330-2337
14 Kaufman M, Singh G, Das S, et al. Efficacy of endoscopic ultrasound-guided celiac plexus block and celiac plexus neurolysis for managing abdominal pain associated with chronic pancreatitis and pancreatic cancer. J Clin Gastroenterol 2010;44(2):127-134

15 Sey MS, Schmaltz L, Al-Haddad MA, et al. Effectiveness and safety of serial endoscopic ultrasound-guided celiac plexus block for chronic pancreatitis. Endosc Int Open 2015;3(1):E56-E59

16 Ascunce G, Ribeiro A, Reis I, et al. EUS visualization and direct celiac ganglia neurolysis predicts better pain relief in patients with pancreatic malignancy (with video) Gastrointest Endosc 2011;73(2):267-274

17 Levy MJ, Gleeson FC, Topazian MD, et al. Combined celiac ganglia and plexus neurolysis shortens survival, without benefit, vs plexus neurolysis alone. Clin Gastroenterol Hepatol 2019;17(4):728-738.e9

18 Santosh D, Lakhtakia S, Gupta R, et al. Clinical trial: a randomized trial comparing fluoroscopy guided percutaneous technique vs. endoscopic ultrasound guided technique of coeliac plexus block for treatment of pain in chronic pancreatitis. Aliment Pharmacol Ther 2009;29(9):979-984

19 Sahai AV, Lemelin V, Lam E, Paquin SC. Central vs. bilateral endoscopic ultrasound-guided celiac plexus block or neurolysis: a comparative study of short-term effectiveness. Am J Gastroenterol 2009;104(2):326-329

20 Alvarez-Sánchez MV, Jenssen C, Faiss S, Napoléon B. Interventional endoscopic ultrasonography: an overview of safety and complications. Surg Endosc 2014;28(3):712-734

21 Davies DD. Incidence of major complications of neurolytic coeliac plexus block. J R Soc Med 1993;86(5):264-266

22 Mittal MK, Rabinstein AA, Wijdicks EF. Pearls \& oysters: acute spinal cord infarction following endoscopic ultrasound-guided celiac plexus neurolysis. Neurology 2012;78(9):e57-e59

23 Bang JY, Sutton B, Hawes RH, Varadarajulu S. EUS-guided celiac ganglion radiofrequency ablation versus celiac plexus neurolysis for palliation of pain in pancreatic cancer: a randomized controlled trial (with videos) Gastrointest Endosc 2019;89(1):58-66.e3

24 Rai P, Cr L, Kc H. Endoscopic ultrasound-guided celiac plexus neurolysis improves pain in gallbladder cancer. Indian J Gastroenterol 2020;39(2):171-175

25 Wang KX, Jin ZD, Du YQ et al. EUS-guided celiac ganglion irradiation with iodine-125 seeds for pain control in pancreatic carcinoma: a prospective pilot study. Gastrointest Endosc 2012;76(5):945-952

26 Kanno Y, Koshita S, Masu K, et al. Efficacy of EUS-guided celiac plexus neurolysis compared with medication alone for unresectable pancreatic cancer in the oxycodone/fentanyl era: a prospective randomized control study. Gastrointest Endosc 2020;92(1):120-130 